\title{
Mitochondrial Delivery Is Essential for Synaptic Potentiation
}

\author{
JAMES JIAYUAN TONG \\ Biophysics and Physiology, University of California, Irvine, California 92697; and Grass Laboratory, \\ Marine Biological Laboratory, 7 MBL Street, Woods Hole, Massachusetts 02543
}

\begin{abstract}
Mitochondria, as portable generators that power synaptic function, regulate the ATP supply and calcium homeostasis in the neuron. As molecular interactions within the synapses before and after the potentiation are beginning to be elucidated, the deciding moment during the tetanic stimulation that gives rise to the strengthening of the synapse remains a mystery. Here, I recorded electrically from an intact Drosophila nervous system, while simultaneously using time-lapse confocal microscopy to visualize mitochondria labeled with green fluorescent protein. I show that tetanic stimulation triggers a fast delivery of mitochondria to the synapse, which facilitates synaptic potentiation. Rotenone, an inhibitor of mitochondrial electron transport chain complex I, suppresses mitochondrial transport and abolishes the potentiation of the synapse. Expression of neurofibromin, which improves mitochondrial ATP synthesis in the neuron, enhances the movements of mitochondria to the synapse and promotes post-tetanic potentiation. These findings provide unprecedented evidence that the mitochondrial delivery to the synapse is critical for cellular learning.
\end{abstract}

\section{Introduction}

Mitochondria play a central role in a variety of cellular processes, including ATP production by oxidative phosphorylation, robust regulation of calcium homeostasis, generation of reactive oxygen species, and initiation of apoptosis (Morris and Hollenbeck, 1993; Wallace, 1999). The neuronal mitochondria vary constantly in their morphology and movement in response to changes in the local energy

Received 15 May 2006; accepted 28 September 2006.

E-mail: tongja@uci.edu

Abbreviations: drp1, dynamin-related protein; EJP, evoked junction potential; GFP, green fluorescent protein; NF1, Neurofibromatosis-1 gene; NMDA, $N$-methyl-D-aspartate; NMJ, neuromuscular junction, PKA, protein kinase A; TMRE, tetramethylrhodamine ethyl ester. state (Nicholls and Budd, 2000; Li et al., 2004; Lisman and Spruston, 2005). Because the soma of the neuron is distant from its terminals, mitochondrial trafficking will substantially shape neuronal function (Chen and Chan, 2006).

The synchronization between mitochondrial conductivity and calcium dynamics in the presynaptic terminal suggests an active role of mitochondria in synaptic plasticity (Jonas et al., 1999). The studies on Milton protein, dynaminrelated protein (drp1), and mitochondrial GTPase dMiro suggest that unimpaired transport and distribution of mitochondria are required for normal synaptic transmission (Stowers et al., 2002; Verstreken et al., 2005; Guo et al., 2005). In the case of drp1, the dependence of reserve-pool vesicular recruitment and mobilization on mitochondria indicates a specific role for mitochondria in regulating synaptic strength (Verstreken et al., 2005). Finally, porin-a building block of the mitochondrial permeability transition pore-functions in both fear conditioning and spatial learning in mice (Weeber et al., 2002) and in the selective up-regulation of mitochondria-encoded genes resulted from learning and memory (Pinter et al., 2005). These findings suggest that changes in mitochondrial function could modulate behavioral plasticity.

Mitochondrial trafficking depends primarily on kinesins for anterograde transport and dyneins for retrograde transport (Goldstein and Yang, 2000). The rapid movements of mitochondria are microtubule-based and the slower movements are actin-based. It is reasonable to envision that mitochondria take an "express train" microtubule to the targeted synapse and employ "local taxi" actin for flexibility in reaching a precise location among the synaptic terminals (Hollenbeck, 1996).

The neuromuscular junction (NMJ) of Drosophila larvae offers an ideal model for studying the role of mitochondrial movements in synaptic plasticity in an intact nervous sys- 
tem because of its organized layout of the motor axons, its transparent muscle fibers, and its conservation of many key synaptic molecules that are also present in mammalian systems (Zhong, 1995; Saitoe and Tully, 2000). As in mammalian central synapses, glutamate is the primary excitatory neurotransmitter at the larval NMJ, with both presynaptic and postsynaptic biochemical signaling contributing to the modulation of synaptic strength. Several forms of synaptic plasticity have been described, including paired-pulse facilitation, short-term facilitation, augmentation, and post-tetanic potentiation - an enhancement of synaptic transmission lasting many minutes after an extensive high-frequency or tetanic presynaptic stimulation (Zucker, 1989). As with memory formation in adult flies, these forms of synaptic plasticity have been dissected by genetic and pharmacological manipulation at the larval NMJ (Zhong, 1995; Saitoe and Tully, 2000; Guo and Zhong, 2006). The NMJ preparation is, therefore, a genetically manipulable and optically accessible system that is useful for studying the role of mitochondria trafficking in synaptic potentiation.

My investigation started by categorizing different populations of mitochondria on the basis of their pattern of movements; recording the effects of motor nerve stimulation on mitochondrial movement and synaptic potentiation, combining time-lapse confocal microscopy with electrophysiological methods; and examining the correlation between the modulation of mitochondrial movements and synaptic potentiation, using rotenone and neurofibromin over-expression.

\section{Materials and Methods}

\section{Drosophila stocks}

Two mitochondrial GFP (mito-GFP) lines were used. The Pilling and Saxton mito-GFP line $W ; D 42-G A L 4$, $p\left\{w^{+m c}=U A S\right.$-mitoGFP $\}$ was generously given by Dr. Pilling and Dr. Saxton at Indiana University (Horiuchi et al., 2005). This line carries a fusion gene with a GAL4-responsive promoter and a mitochondrial import sequence fused to GFP, and also a D42-GAL4 promoter that produces motorneuron-specific expression of this mitochondrial marker. The Cox mito-GFP line, which is constructed by fusing the human COX VIII mitochondrial targeting signal to the $\mathrm{N}$ terminus of enhanced green fluorescent protein (Cox and Spradling, 2003), was generously given to me by Dr. Rachel Cox from the Carnegie Institution of Washington. In the offspring from the cross between the Cox mito-GFP line and the ELAV-GAL4 line (Bloomington Stock center), the neuronal mitochondria in the larva are labeled. Most of the data in this study were collected with the Pilling and Saxton mito-GFP line, which produces a stronger signal.

The $h s N F 1 /+$ line (also called $h s N F 1 / m i t o-G F P$ ) was obtained from the cross between $h s N F 1 / h s N F 1$; + flies and the Pilling and Saxton mito-GFP line. The motor neurons of the hsNF1/mito-GFP flies express mito-GFP and neurofibromin by the leakage expression of a heat-shock-controlled Neurofibromatosis-1 (NF1) transgene (Guo et al., 2000; Tong et al., 2007).

The CD8-GFP line was used to visualize the branching of the synaptic terminals at the neuromuscular junction (NMJ). The line was a gift from Dr. Mani Ramaswami at the University of Arizona, Tucson.

\section{Neuromuscular junction preparation}

Drosophila 3rd instar larvae were mounted in a recording chamber under Schneider solution (Sigma) perfusion at $25^{\circ} \mathrm{C}$ to be dissected dorsally to expose the ventral nerve cord. Data were collected from synaptic terminals that innervate muscle fibers $6,7,12$, and 13 . To co-stain synaptic mitochondria, the preparations were incubated for $10 \mathrm{~min}$ at $25^{\circ} \mathrm{C}$ with tetramethylrhodamine ethyl ester (TMRE, 10 nmol $1^{-1}$ ), a membrane-sensitive mitochondrial dye.

\section{Image acquisition and analysis}

Images were acquired by a Zeiss upright LSM510 META confocal microscope with both $40 \times($ NA 1.2) and $63 \times($ NA 1.4) water immersion objectives. For time-lapse imaging, confocal images of NMJ were collected at $0.5-\mu \mathrm{m}$ steps throughout the entire $z$ dimension, which takes about $1 \mathrm{~min}$ for the collection of the entire stack. The $40 \times$ lens was used in most experiments. Confocal images were then collapsed in the $z$-axis to yield two-dimensional projections. The movements of mitochondria were traced and measured manually at individual NMJ by MetaMorph software, ver. 7 (Universal Imaging). The recruitment of mitochondria to the terminals under tetanic stimulation was analyzed by a multi-object tracking paradigm customized in Metamorph software with manual confirmation. To analyze the redistribution of mitochondria at the synapse, mitochondrial density was recorded by measuring the number of mitochondria per micrometer at given time points during the recording. Statistical analysis was performed with Graphpad Prism software, ver. 4, and the Microsoft Excel statistical analysis.

\section{Electrophysiology}

The nerve terminals were stimulated by electric pulses (1 ms, 2 times threshold voltage) delivered to the appropriate segmental nerve at $10 \mathrm{~Hz}$ via a glass suction electrode (20- $\mu \mathrm{m}$ inside diameter). Synaptic potentiation was recorded and measured using a protocol adapted from Kuromi and Kidokoro (2000). In rotenone inhibition experiments, rotenone $\left(1 \mu \mathrm{mol} \mathrm{l}^{-1}\right)$ was applied through the suction electrode that bathed the motor axon. Electrophysiological recordings were made along with the image acquisition. 


\section{Mitochondrial respiration and ATP synthesis rate}

Mitochondria were isolated from adult flies of two lines: hsNFll+ (experimental) and mito-GFP (controls). In each case, 40 to 80 flies were gently crushed in a $10-\mathrm{ml}$ Kontes homogenizer with seven strokes of the pestle in $3 \mathrm{ml}$ of homogenization buffer consisting of $225 \mathrm{mmol} \mathrm{l}^{-1}$ mannitol, $75 \mathrm{mmol} \mathrm{l}^{-1}$ sucrose, $10 \mathrm{mmol} \mathrm{l}^{-1} 3$-( $N$-morpholino) propane sulfonic acid, $1 \mathrm{mmol} \mathrm{l}^{-1}$ ethylene glycol tetraacetic acid, and $0.5 \%$ bovine serum albumin, $\mathrm{pH} 7.2$, at $4^{\circ} \mathrm{C}$. The extracts were filtered through eight layers of cheesecloth, and then centrifuged in a Beckman Avantis-J25 at $300 \times g$ for $3 \mathrm{~min}$. The supernatant was centrifuged at $6000 \times g$ for $10 \mathrm{~min}$ to obtain a mitochondrial pellet. Mitochondrial protein was determined by the Bradford method, using Bio-Rad reagents; the values were corrected for the bovine serum albumin in the homogenization buffer. Respiration rates were determined by oxygen consumption using a Clark-type electrode and metabolic chamber containing $650 \mu \mathrm{l}$ of reaction buffer consisting of $225 \mathrm{mmol}^{-1}$ mannitol, $75 \mathrm{mmol}^{-1}$ sucrose, $10 \mathrm{mmol} \mathrm{l}^{-1} \mathrm{KCl}, 10 \mathrm{mmol}$ $1^{-1}$ Tris- $\mathrm{HCl}$, and $5 \mathrm{mmol}^{-1} \mathrm{KH}_{2} \mathrm{PO}_{4}, \mathrm{pH} 7.2$, at $25^{\circ} \mathrm{C}$. Rates of ATP synthesis were calculated on the basis of ADP consumption and amount of oxygen consumption during mitochondrial respiration (Tong et al., 2007).

Rotenone $\left(1 \mathrm{mmol} \mathrm{l}^{-1}\right)$ was added to the isolated mitochondria in the metabolic chamber to measure the inhibition of ATP synthesis to be compared with untreated mitochondria as controls.

\section{Results}

Microanatomy of the motor axons, their synapses, and mitochondria

Mitochondria were genetically labeled with mito-GFP expressed in motor neurons of Drosophila larvae with motor-neuron-specific D42-Gal4 driver (methods). The threedimensional stacked, time-lapse confocal microscopy resolves individual mitochondria (longitudinal axis: 0.3-1.2 $\mu \mathrm{m})$ and tracks their movements at different optical sections. TMRE, a membrane-sensitive mitochondrial dye, was used to co-stain synaptic mitochondria (Fig. 1a-c).

\section{Categories of mitochondrial populations}

Synaptic mitochondria can be categorized into two populations on the basis of their distinctive movement patterns. One is the "resident pool," which consists of mitochondria that reside at the synaptic terminal and show limited movements. The other population is the "activated pool," which consists of mitochondria that move to or from the terminal during the recording (see Movie 1 at http://www.biolbull. org/supplemental/). The percentage of activated mitochondria within the total terminal population was $21.4 \% \pm \mathrm{SD}$ $2.3 \%$ ( $n=17$ terminals from 9 larvae). Among the activated mitochondria, $51.2 \% \pm 2.6 \%$ showed anterograde movement toward the synaptic terminal, and $49.4 \% \pm 3.1 \%$ showed retrograde movement away from the terminal $(P=$ 0.14 comparing anterograde and retrograde mitochondria).

\section{Motor nerve stimulation increases mitochondrial density at the synapse in potentiation}

I used a combination of time-lapse confocal mitochondrial tracking and dual sharp-electrode voltage-clamp technique to record evoked junction potential (EJP) at the NMJ (Fig. 1d). Tetanic stimulation $(10 \mathrm{~Hz})$ of the motor nerve increased the delivery of mitochondria to the synapse (Fig. 2a; Movie 2 at http://www.biolbull.org/supplemental/). The immediate bifurcation point (20-30 $\mu \mathrm{m}$ upstream from the synapse) was chosen as the starting point for recording the movements of mitochondria. In response to tetanic stimulation, mitochondrial density was significantly increased at the stimulated synapses while mitochondrial density in control synapses remained steady throughout the experiment. The potentiation of the synapse, demonstrated as an increase in EJP, followed the accumulation of mitochondria at the synapse (Fig. 2a).

\section{Actions of rotenone and neurofibromin}

Effects on the respiration of isolated mitochondria. Application of a potent complex I inhibitor, rotenone $(1 \mu \mathrm{mol}$ $1^{-1}$ ), in the suction electrode that bathed the projecting axon loop (Fig. 2) blocked both the delivery of mitochondria to the synapse and the potentiation of the synapse (Fig. 2). Neurofibromin expression by $h s N F 1$ transgene increased mitochondrial respiration by enhancing complex I activity (Tong et al., 2007), which increases the mitochondrial ATP production rate by $54 \%$ (Fig. 3 ).

Effects of neurofibromin on mitochondrial movements and on synaptic potentiation. Neurofibromin expression did not alter the density of mitochondria in the NMJ synapses before tetanic stimulation of the preparation (Fig. 2a); however, in response to stimulation, neurofibromin expression in these neurons promoted a fast elevation of the synaptic mitochondrial density. The mitochondrial density in elevated neurofibromin terminals was significantly higher than that in the control synapses that are potentiated (Fig. 2a). EJP amplitudes in synapses with neurofibromin over-expression were also increased above those of potentiated control synapses (Fig. 2a). Moreover, after the initiation of tetanus stimulation, both mitochondrial accumulation and synaptic potentiation occurred $5 \mathrm{~min}$ earlier in synapses over-expressing neurofibromin than in control synapses (Fig. 2). 

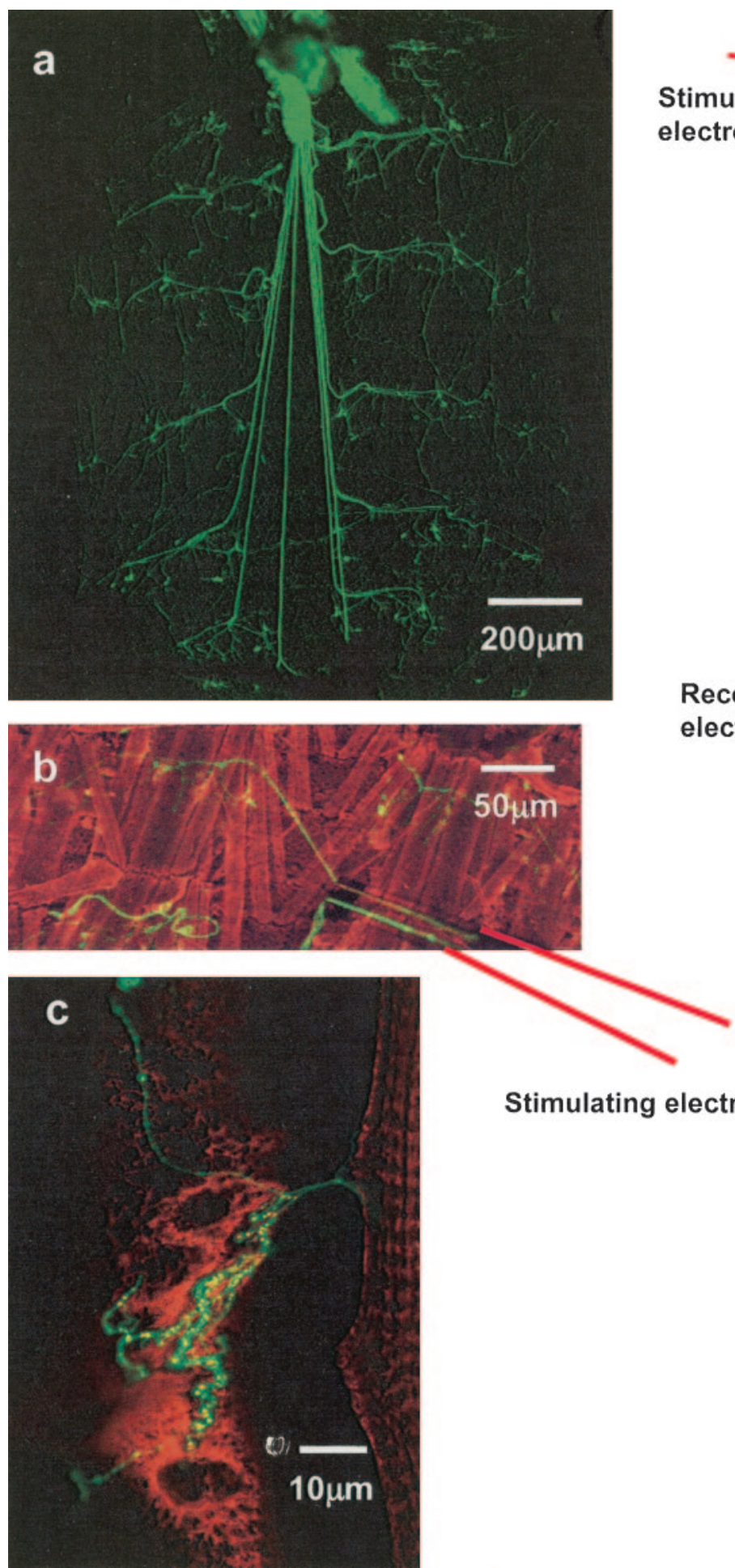

\section{Stimulating electrode}

\section{anterograde}

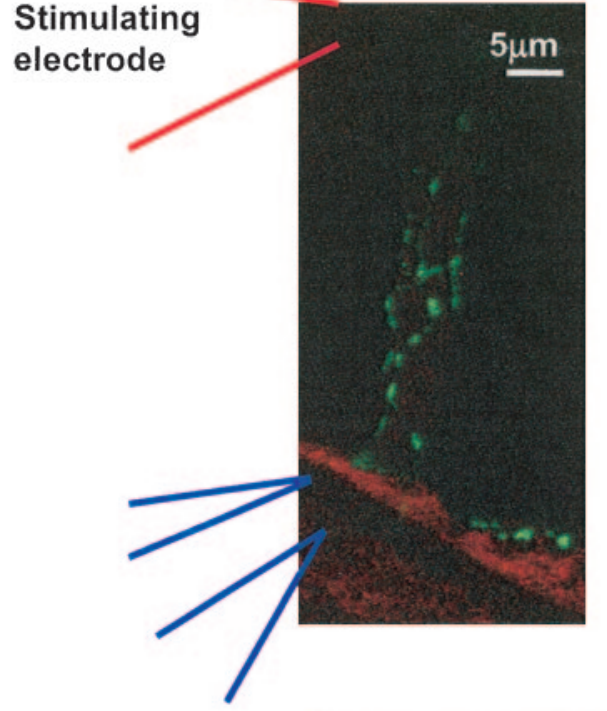

1

d

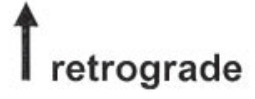

Figure 1. Microanatomy of motor axons, synaptic terminals, and mitochondrial movements under synaptic stimulation. (a) Drosophila larval motor nerves labeled by expression of motor-neuron-specific mito-GFP. (b) Suction electrode (red) is positioned to stimulate projecting axon loop of the motor nerve innervating the synaptic terminal. This suction electrode was also used to apply rotenone to the axons. Muscle fibers have a salmon color in the presence of TMRE. (c) Mitochondrial distribution (bright orange dots, TMRE labeled) in the synaptic terminals of CD8 GFP line flies (green) branching over muscle fibers at NMJ. (d) GFP-labeled neuronal mitochondria at the presynaptic terminal with schematic arrangement of stimulation and recording electrodes. The movements of individual mitochondria are indicated at $15 \mathrm{~min}$ and $19 \mathrm{~min}$, the yellow and orange arrows identify two anterograde mitochondria, and the red arrow marks one retrograde mitochondria (see Movie 2 at http://www.biolbull.org/supplemental/). 


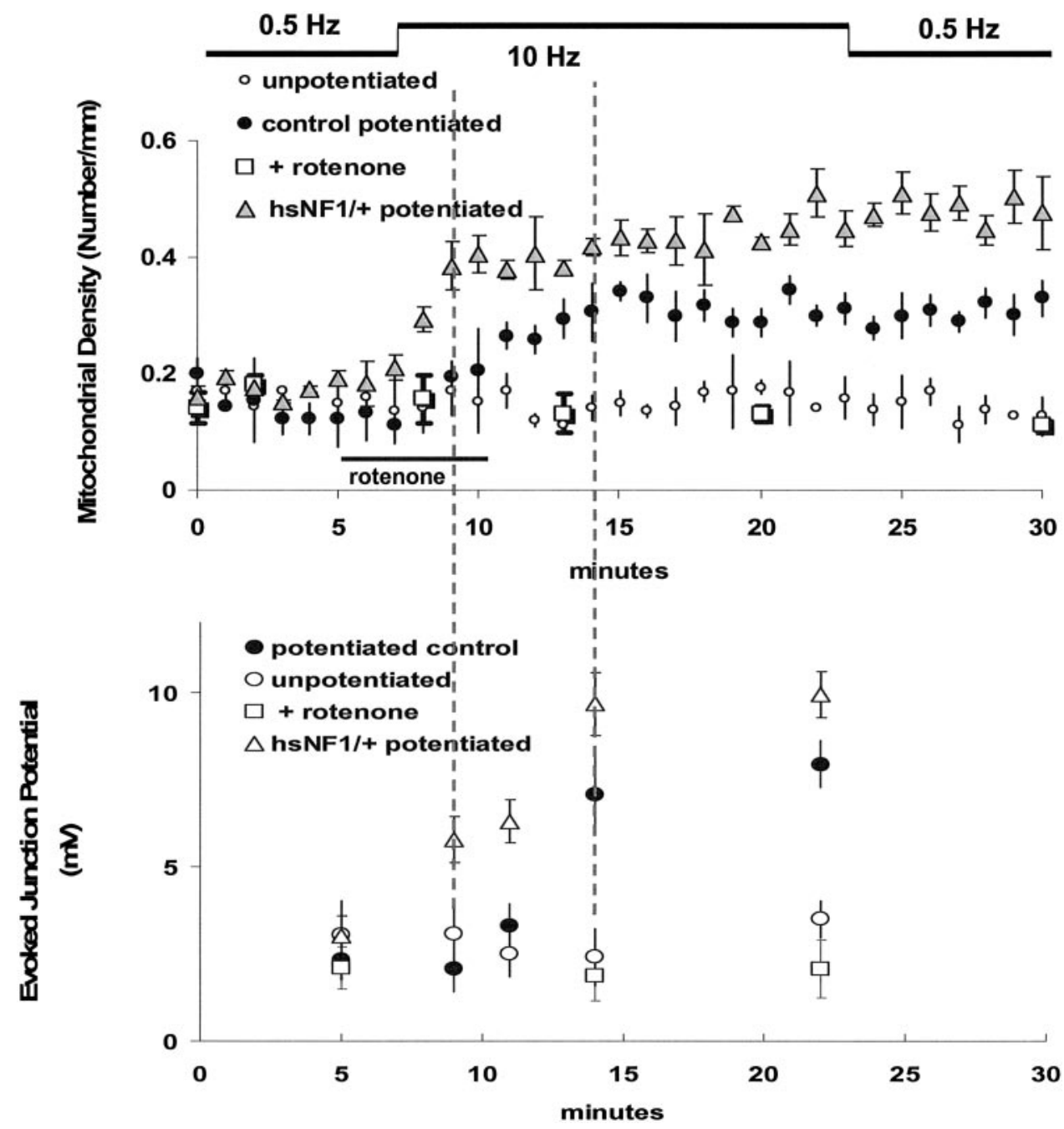

Figure 2. Correlation between mitochondrial movements and synaptic potentiation. Upper panel: Mitochondrial density (M.D.) is the number of mitochondria per micrometer measured because the diameter of nerve branches at the NMJ presynaptic terminal appears to be constant. The density is presented in terms of length rather than volume. M.D. of the control-potentiated terminal $(-)$ is compared with the section-matching contralateral unpotentiated terminal $(\bigcirc)$. Density increase indicates a net increase of mitochondrial flow toward the synapse. Data points are means $\pm \mathrm{SD}(n=8$ terminals). There is no significant difference before $10 \mathrm{~min}(P$ $=0.13-0.21)$. Significant difference between unpotentiated and potentiated synapses is detected after $10 \mathrm{~min}$ $(P<0.001)$. Lower panel: Evoked synaptic potentials (EJPs) were measured and plotted. The M.D. and EJP measurements under rotenone $\left(1 \mu \mathrm{mol} 1^{-1}\right)$ are shown $(\square)$ with the bar $(\longrightarrow)$ indicating the application of rotenone via suction electrode from $5 \mathrm{~min}$ until $10 \mathrm{~min}$ for both panels. Significant differences were detected between control and potentiated EJP amplitude at $14 \min (P<0.001)$ and $22 \min (P<0.001)$. The dashed lines indicate the timing between potentiation occurrence and mitochondrial density increase, comparing control synapses to neurofibromin expression synapses. Neurofibromin enhanced the delivery of mitochondria to the synaptic terminals, demonstrated as a significant elevation of mitochondrial density during tetanic stimulation, correlated with an increased EJP in the $h s N F 1 /+$ synapses $(\mathbf{\Delta})$ at 9, 11, 14, and 22 min compared to both the unpotentiated $(P<0.001)$ and control potentiated synapses $(P<0.01$ at 9 and $11 \mathrm{~min}, P<0.02$ for at 14 and $22 \mathrm{~min}$ ). Stimulation: $1 \mathrm{~ms}, 2 \times$ threshold, $10 \mathrm{~Hz}$; begins at $7 \mathrm{~min}$, ends at $23 \mathrm{~min}$. Data collected each minute.

\section{Discussion}

This study demonstrates, for the first time in an intact nervous system, that tetanic stimulation triggers the delivery of mitochondria to specifically targeted synapses, and that the resulting reorganization of the mitochondrial network may be a prerequisite for synaptic potentiation. The analysis of mitochondrial movement indicates that the net increase in mitochondrial density seems to be mainly due to an increase in the rate of mitochondrial anterograde movement towards 


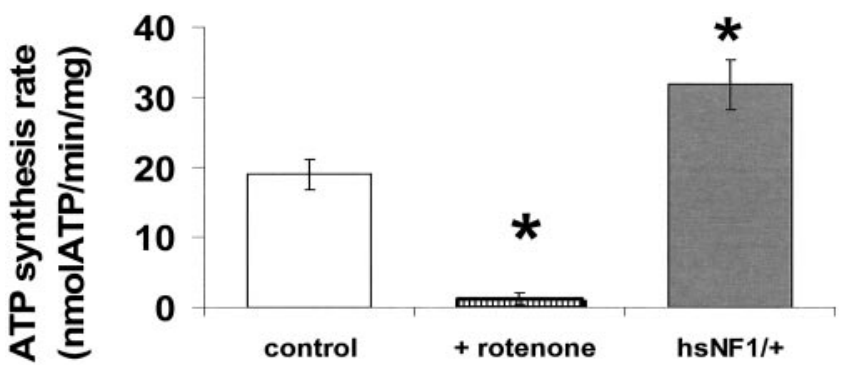

Figure 3. Mitochondria isolated from $h s N F 1 /+$ flies have a higher rate of ATP production than control. Rotenone $\left(1 \mu \mathrm{mol} \mathrm{l}^{-1}\right)$ added to $h s N F 1 /+$ mitochondria reduced the rate of ATP production. Asterisk (*) indicates that rates are significantly different from control mitochondria isolated from mito-GFP lines, $P<0.01, n=5$.

the synapse. The unprecedented finding that the synapse is potentiated when mitochondrial dynamics are elevated by neurofibromin expression, which leads to an increased ATP production, suggests a mitochondrial approach to the enhancement of learning and memory.

The physiological significance of anterograde and retrograde mitochondria invites further scrutiny. Although the retrograde mitochondria may be recycled or degraded after their service at the synapse, the anterograde mitochondria may accommodate the energy demand of synaptic potentiation. These newly delivered mitochondria may remodel the calcium homeostasis and the energy capacity required to sustain the potentiated synaptic activity, with possible preparation for long-term modification of the synapse. Genetic marking of an individual anterograde or retrograde mitochondrion will provide further insight into the destination of these mitochondria and their active roles in shaping the function of their host neuron.

The proximate signal that triggers the increased rate of mitochondrial delivery to the activated synapses remains to be elucidated. In determining whether the insertion of mitochondria into the synapse is sufficient to initiate synaptic potentiation, the challenge will be to manipulate the mitochondria into a silent synapse without using electric stimulation and then test whether synaptic strengthening occurs.

There is tremendous potential to employ these real-time in vivo imaging analyses that investigate the synchronicity of neuronal and behavioral dynamics at both the cellular and behavioral levels as a means to characterize phenotypes of biomedical interests (Bokil et al., 2006; Misgeld and Kerschensteiner, 2006).

The Neurofibromatosis-1 gene (NF1) regulates the adenylyl cyclase and protein kinase A (AC/PKA) pathway and Ras signaling (Guo et al., 2000; Tong et al., 2002). The over-expression of neurofibromin increases ATP production by elevating complex I activity through PKA phosphorylation of complex I subunits (Papa, 2002; Tong et al., 2007). Flies with neurofibromin over-expression also exhibit prolonged olfactory memory retention (unpubl. data). Further experiments are warranted to investigate whether mitochondrial dynamics are active in several learning and memoryenhancement manipulations such as the expression of cAMP-responsive element-binding protein (CREB) in Drosophila (Yin et al., 1995) and the over-expression of adenylyl cyclase (Wang et al., 2004) and NMDA receptor 2B (Tang et al., 1999) in mice.

Mitochondrial dynamics, in a more general sense, encompasses mitochondrial functional fluctuation, movements, fusion and fission of individual mitochondria, and reorganization of the mitochondrial network. If the unique temporal and spatial dynamic patterns of mitochondrial distribution are a "mitochondrial memory code" that dictates the potentiation of specific synapses, the plasticity of the neuronal network, and ultimately a decisive modification of behavior, the disruption of the "code"-manifested as functional aberration and movement disruption - will inevitably result in an energy imbalance, loss of homeostasis, and deficiency of synaptic plasticity that underlie the cognitive deterioration seen in pathological conditions and aging.

\section{Acknowledgments}

I greatly appreciate Dr. William Saxton and Dr. Aaron Pilling for sending their neuronal mito-GFP line, Dr. Rachel Cox for sending her UAS-mitoGFP lines, and Dr. Mani Ramaswami for sending his CD8 GFP line. Acknowledgments also go to Dr. Elizabeth Jonas, Dr. Vincent J. Caiozzo, Dr. Samuel E. Schriner, Dr. Shirley J. Gaffey, and Dr. Douglas C. Wallace. This project is supported by Grass fellowship awards $(2004,2006)$ to J.T. and NIH funding AR47752-01A1.

\section{Literature Cited}

Bokil, H., O. Tchernichovsky, and P. P. Mitra. 2006. Dynamic phenotypes: time series analysis techniques for characterizing neuronal and behavioral dynamics. Neuroinformatics 4: 119-128.

Chen, H., and D. C. Chan. 2006. Critical dependence of neurons on mitochondrial dynamics. Curr. Opin. Cell Biol. 18: 453-459.

Cox, R. T., and A. C. Spradling. 2003. A Balbiani body and the fusome mediate mitochondrial inheritance during Drosophila oogenesis. Development 130:1579-1590.

Goldstein, L. S., and Z. Yang. 2000. Microtubule-based transport systems in neurons: the roles of kinesins and dyneins. Annu. Rev. Neurosci. 23: 39-71.

Guo, H. F., and Y. Zhong. 2006. Requirement of Akt to mediate long-term synaptic depression in Drosophila. J. Neurosci. 26: 4004 4014

Guo, H. F., J. Tong, F. Hannan, L. Luo, and Y. Zhong. 2000. A neurofibromatosis-1-regulated pathway is required for learning in Drosophila. Nature 403: 895-898.

Guo, X., G. T. Macleod, A. Wellington, F. Hu, S. Panchumarthi, M. Schoenfield, L. Marin, M. P. Charlton, H. L. Atwood, and K. E. Zinsmaier. 2005. The GTPase dMiro is required for axonal transport of mitochondria to Drosophila synapses. Neuron 47: 379-393.

Hollenbeck, P. J. 1996. The pattern and mechanism of mitochondrial transport in axons. Front. Biosci. 1: 91-102. 
Horiuchi, D., R. V. Barkus, A. D. Pilling, A. Gassman and W. M. Saxton. 2005. APLIP1, a kinesin binding JIP-1/JNK scaffold protein, influences the axonal transport of both vesicles and mitochondria in Drosophila. Curr. Biol. 15: 2137-2141.

Jonas, E. A., J. Buchanan, and L. K. Kaczmarek. 1999. Prolonged activation of mitochondrial conductances during synaptic transmission. Science 286: 1347-1350.

Kuromi, H., and Y. Kidokoro. 2000. Tetanic stimulation recruits vesicles from reserve pool via a cAMP-mediated process in Drosophila synapses. Neuron 27: 133-143.

Li, Z., K. Okamoto, Y. Hayashi, and M. Sheng. 2004. The importance of dendritic mitochondria in the morphogenesis and plasticity of spines and synapses. Cell 119: 873-887.

Lisman, J., and N. Spruston. 2005. Postsynaptic depolarization requirements for LTP and LTD: a critique of spike timing-dependent plasticity. Nat. Neurosci. 8: 839-841.

Misgeld, T., and M. Kerschensteiner. 2006. In vivo imaging of the diseased nervous system. Nat. Rev. Neurosci. 7: 449-463.

Morris, R. L., and P. J. Hollenbeck. 1993. The regulation of bidirectional mitochondrial transport is coordinated with axonal outgrowth. J. Cell Sci. 104: 917-927.

Nicholls, D. G., and S. L. Budd. 2000. Mitochondria and neuronal survival. Physiol. Rev. 80: 315-360.

Papa, S. 2002. The NDUFS4 nuclear gene of complex I of mitochondria and the cAMP cascade. Biochim. Biophys. Acta 1555: 147-153.

Pinter, M., D. D. Lent, and N. J. Strausfeld. 2005. Memory consolidation and gene expression in Periplaneta americana. Learn. Mem. 12: $30-38$.

Saitoe, M., and T. Tully. 2000. Making connections between developmental and behavioral plasticity in Drosophila. Chapter 15 in Toward a Theory of Neuroplasticity, C. A. Shaw and J. C. McEachern, eds. Psychology Press, Philadelphia.

Stowers, R. S., L. J. Megeath, J. Gorska-Andrzejak, I. A. Meinertzhagen, and T. J. Schwarz. 2002. Axonal transport of mitochondria to synapses depends on Milton, a novel Drosophila protein. Neuron 36: 1063-1077.

Tang, Y. P., E. Shimizu, G. R. Dube, C. Rampon, G. A. Kerchner, M. Zhuo, G. Liu, and J. Z. Tsien. 1999. Genetic enhancement of learning and memory in mice. Nature 401: 63-69.

Tong, J., F. Hannan, Y. Zhu, A. Bernards, and Y. Zhong. 2002. Neurofibromin regulates $\mathrm{G}$ protein-stimulated adenylyl cyclase activity. Nat. Neurosci. 5: 95-96.

Tong, J. J., S. E. Schriner, D. McCleary, B. J. Day, and D. C. Wallace. 2007. Life extension through neurofibromin mitochondrial regulation and antioxidant therapy for neurofibromatosis-1 in Drosophila melanogaster. Nat. Genetics 39: 476-485.

Verstreken, P., C. V. Ly, K. J. Venken, T. W. Koh, Y. Zhou, and H. J. Bellen. 2005. Synaptic mitochondria are critical for mobilization of reserve pool vesicles at Drosophila neuromuscular junctions. Neuron 47: 365-378.

Wallace, D. C. 1999. Mitochondrial diseases in man and mouse. Science 283: $1482-1488$

Wang, H., G. D. Ferguson, V. V. Pineda, P. E. Cundiff, and D. R. Storm. 2004. Overexpression of type-1 adenylyl cyclase in mouse forebrain enhances recognition memory and LTP. Nat. Neurosci. 7: 635-642.

Weeber, E. J., M. Levy, M. J. Sampson, K. Anflous, D. L. Armstrong, S. E. Brown, J. D. Sweatt, and W. J. Craigen. 2002. The role of mitochondrial porins and the permeability transition pore in learning and synaptic plasticity. J. Biol. Chem. 277: 18891-18897.

Yin, J. C., M. Del Vecchio, H. Zhou, and T. Tully. 1995. CREB as a memory modulator: induced expression of a dCREB2 activator isoform enhances long-term memory in Drosophila. Cell 81: 107-115.

Zhong, Y. 1995. Mediation of PACAP-like neuropeptide transmission by coactivation of Ras/Raf and cAMP signal transduction pathways in Drosophila. Nature 375: 588-592.

Zucker, R. S. 1989. Short-term synaptic plasticity. Annu. Rev. Neurosci. 12: $13-31$. 